Bekanntheit von IPP in der produzierenden Wirtschaft Bayerns

\title{
Spezialistenthema mit Potenzial
}

\author{
Mit Integrierter Produktpolitik wurde ein neues Konzept in die Umweltdiskus- \\ sion eingeführt. Alle Akteure, von der Politik über Hersteller und Handel bis \\ hin zum Konsumenten sind nun aufgerufen, IPP mit Leben zu füllen. Aber erst \\ dann, wenn IPP bekannt und auch verstanden wird, ist mit einer nennens- \\ werten Umsetzung in praktisches Handeln zu rechnen.
}

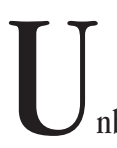
nbestritten zählt die produzierende Wirtschaft zu den wichtigsten Akteuren bei der Umsetzung von Integrierter Produktpolitik (IPP). Aus diesem Grunde wurde das Bayerische Institut für Angewandte Umweltforschung und -technik GmbH (BifA) durch das Bayerische Staatsministerium für Umwelt, Gesundheit und Verbraucherschutz beauftragt, die Bekanntheit und den Umsetzungsgrad von IPP im bayerischen produzierenden Gewerbe zu untersuchen. In diesem Beitrag werden wichtige Ergebnisse der Erstuntersuchung aus dem Jahr 2001 sowie der ersten Wiederholung im Jahr 2003 dargestellt.

Als quantitative Untersuchungsmethode wurde eine repräsentative Telefonbefragung gewählt. Die Entwicklung des hierzu verwendeten Fragebogens erfolgte unter Einsatz qualitativer Methoden, nämlich einer Gruppendiskussion mit ausgewiesenen IPP-Experten sowie Interviews mit der so genannten Repertory-Grid-Methode.

Interviews wie Gruppendiskussion zeigten, dass IPP ein komplexer, noch nicht klar positionierter Begriff ist, der von vielen Befragten sogar als recht diffus und sperrig empfunden wird. Ein Experte sagte: ,IPP wird nur sehr langsam bei den am Wirtschaftprozess Beteiligten in Fleisch und Blut übergehen.“

\section{Wer kennt IPP?}

Die beiden für die produzierende Wirtschaft Bayerns repräsentativen Telefonbefragungen mit 380 Betrieben im Jahr 2003 beziehungsweise $370 \mathrm{im}$ Jahr 2001 zeigten, dass in der neuen Umfrage 25 Prozent der Befragten (2001: 27,6 Prozent) von IPP schon etwas gehört hatten. Aber nur 12,1 Prozent (2001: 16,2 Prozent) konnten auf Nachfrage auch konkretere Vorstellungen zu dem Begriff äußern. Als „IPP-Kenner“ wurden solche Befragte definiert, die von IPP schon gehört hat- ten und gleichzeitig in der Lage waren, dezidierte Begriffserläuterungen vorzunehmen. Diese Erläuterungen bezogen sich in der Erstuntersuchung vornehmlich auf einige wenige, sich zum Teil überlappende Bereiche, wobei aber 2003 viele dieser Erklärungen von IPP schon deutlich präziser erschienen als noch 2001.

So beziehen sich zwar nach wie vor 45 Prozent der Nennungen auf das Thema „Ganzheitlichkeit“, womit vor allem „umfassende“, „kreislaufartige“ oder „Lebenszyklus spezifische“ Betrachtungen von Produkten oder Produktionsprozessen gemeint waren. Diese Nennungen waren aber oftmals viel konkreter als dies noch vor zwei Jahren der Fall war. Zwei Beispiele mögen dies veranschaulichen. Ein Befragter antwortete: „Ich bin selbst Mitglied des Arbeitskreises IPP in unserer Firma. IPP meint den gesamten Produkt-Lebenszyklus. Das heißt, von der Auswahl der Rohstoffe, über Verpackung, Nutzung und Entsorgung; es wird betrachtet, was umweltmäßig das Beste ist." Ein anderer sagte: „IPP ist die ganzheitliche Betrachtung des Produktes, ,von der Wiege bis zur Bahre'; es werden alle Faktoren wie zum Beispiel Energie oder Abfall betrachtet".

Darüber hinaus ergab die Wiederholungsbefragung, dass die Unternehmen zum Teil sogar erste IPP-Strategien etablieren konnten: „Unsere Philosophie und innerbetriebliche Strategie ver- folgt das Ziel, unter Umweltgesichtspunkten den Verbraucher, also den Nutzer, und die Wirtschaftlichkeit (des Produktionsprozesses) unter einen Hut zu bringen."

Gleichwohl wurde auch deutlich, dass der Begriff bisweilen auch recht kritisch eingeschätzt wird: „IPP ist ein abgedroschener, schwammiger Begriff; schlecht interpretierbar. Es ist eigentlich ein altes Instrument, das einen neuen Namen erhalten hat. Für mich bedeutet IPP saubere Unternehmensfiuhrung".

\section{Wer kennt IPP wirklich?}

Um den Kenntnisstand der IPP-Kenner von der Bedeutung des Begriffs noch differenzierter einschätzen zu können, wurde 2003 eine zusätzliche Itembatterie entwickelt. Den Ausgangspunkt bildete der inzwischen in gewissem Umfange gegebene ,common sense“ bezüglich der Begriffsinterpretation (Abb. 1).

Diejenigen Befragten, die nähere Angaben zur Bedeutung von IPP machen konnten, wurden zusätzlich gefragt, welche Aussagen einer zuvor umfänglich getesteten Fragen-Batterie sie als Kernelemente des IPP-Konzeptes ansehen würden. Auf diese Weise konnte die „Tiefe“ des IPP-Kenntnisstandes noch deutlicher zu Tage treten. Bezogen auf die Gesamtstichprobe zeigte sich, dass die 12,1 Prozent „IPP-Kenner“ den Begriff nicht gleichermaßen erfassen: nur 3,9 Prozent sind „sehr gute IPP-Kenner“. Weitere 7,4 Prozent kennen IPP ,überdurchschnittlich gut“, und 0,8 Prozent sind ,unterdurchschnittlich gute Kenner“. Der Begriff IPP bleibt damit auch im Jahr 2003 der großen Mehrheit unbekannt. Der Bekanntheitsgrad ist sogar geringfiugig zurückgegangen. Diejenigen Befragten, die wir als „IPP-Kenner“ bezeichnen, erwiesen sich aber zum weitaus größten Teil als überdurchschnittlich gute oder sehr gute IPP-Kenner. Es ist also offensichtlich gelungen, den Begriff bei einem sehr großen Teil der „IPPKenner" gut zu verankern. Und sicherlich ist dieser Erfolg für die Verbreitung des IPP-Gedankens

\begin{tabular}{|c|c|c|}
\hline \multicolumn{3}{|c|}{ Gegenstand: Produkte und Dienstleistungen } \\
\hline \multicolumn{3}{|c|}{ ZIEL: Senkung der Umweltbelastungen öber die gesamte Lebenswegkette } \\
\hline \multicolumn{3}{|c|}{ STRATEGIE: Integration } \\
\hline $\begin{array}{l}\text { Der gesamte } \\
\text { Produktlebensweg }\end{array}$ & $\begin{array}{l}\text { Kommunikation } \\
\text { \& Kooperation }\end{array}$ & $\begin{array}{l}\text { Ökologie } \\
\text { \& Ökonomie }\end{array}$ \\
\hline $\begin{array}{l}\text { Einschlieflich Entwicklung, } \\
\text { Marketing, Vertrieb, Nutzung ... }\end{array}$ & $\begin{array}{c}\text { Hersteller, Politik, Handel, Konsumenten, } \\
\text { Marketing, Vertrieb ... }\end{array}$ & $\begin{array}{l}\text { Mit Blick auf alle Umweltwirkungen } \\
\text { Über die Grenzen des Unternehmens hinaus }\end{array}$ \\
\hline
\end{tabular}


von wesentlich größerer Bedeutung, als es eher geringfügige Änderungen des Anteils derjenigen Befragten sind, die mit IPP allenfalls diffuse Vorstellungen verbinden.

\section{- Thema für Spezialisten}

Während nur etwa jeder zwanzigste Befragte aus kleinen Unternehmen mit dem IPP-Begriff etwas anzufangen weiß, ist es bei den mittleren Unternehmen etwa jeder zehnte. Bei den großen Unternehmen mit mehr als 500 Mitarbeitern sind es immerhin rund 40 Prozent der Befragten. Und während es in den Umweltabteilungen 18 Prozent Kenner gibt und in Geschäftsfuihrungen und Vorständen immerhin noch 15,1 Prozent, ist der Begriff in Marketing und Vertrieb vollständig unbekannt.

Der Begriff ist also weniger als erhofft in breite Kreise der Wirtschaft diffundiert - obwohl er von seinem Bedeutungsgehalt her durchaus großes Potenzial hätte. Vielmehr bleibt er Experten vorbehalten, also Spezialisten aus größeren Unternehmen und hier vor allem aus dem Umwelt- und Abfallbereich sowie der Geschäftsleitung.

Auf die Frage, ob IPP für ihr Unternehmen interessant sei, antworteten 2001 etwa 40 Prozent der „Kenner“ - also derjenigen Personen, die auch auf Nachfrage nähere Angaben zur Begriffsbedeutung machen konnten - mit ,ja“, 36 Prozent fanden IPP ,zum Teil interessant“, aber 23 Prozent zeigten „kein Interesse“. 2003 wird IPP von etwa drei Vierteln zumindest teilweise für interessant befunden - ein Viertel zeigte sich desinteressiert. Von den Befragten, die sich für IPP zumindest teilweise interessieren, setzen immerhin 31,4 Prozent IPP regelmäßig und 43,5 Prozent gelegentlich ein. Fragt man nach den Erfahrungen und Erwartungen dieser „IPP-Anwender“, so ergibt sich auch im Jahr 2003 wieder ein ausgesprochen positives Bild (Abbildung 2).

Immerhin 41 Prozent der Anwender erwarten mittel- bis langfristig eine Kostensenkung in ihrem Unternehmen durch IPP (2001: 38,7 Prozent ); über 70 Prozent gehen davon aus, dass IPP mittelbis langfristig die Marktchancen des eigenen Unternehmens verbessern wird (2001: 67,6 Prozent). Schließlich erwarten knapp 90 Prozent eine Entlastung der Umwelt durch IPP (2001: knapp 80 Prozent ). IPP führt also in der Wahrnehmung der Anwender kurzfristig zu keinen greifbaren Ergebnissen, gleichwohl wird IPP als eine außerordentlich erfolgreiche mittel- bis langfristige Strategie verstanden.

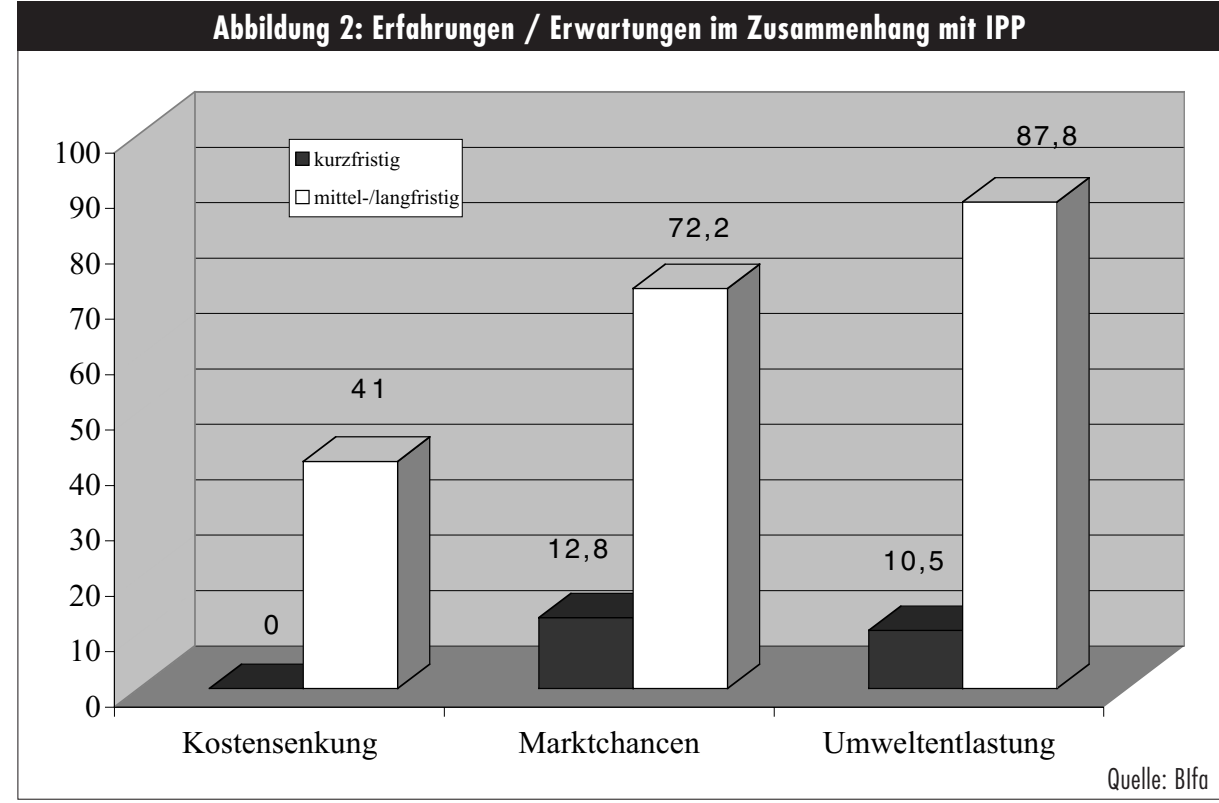

\section{Hohe Resonanz bei Kernaussagen}

In beiden Untersuchungen fanden einzelne IPPKernaussagen bemerkenswert hohe Zustimmung, und zwar selbst von Befragten, die bislang noch keinerlei Bezug zu IPP hatten. Dies macht deutlich, dass das Konzept der Integrierten Produktpolitik ausgesprochene „Highlights“ bietet, denen spontan nahezu jeder zustimmt.

Stärkste Resonanz erfuhren die Statements offenbar dann, wenn nachhaltige, ganzheitliche oder kooperative Lösungsansätze zur Disposition standen: So teilten über 80 Prozent aller Befragten die Meinung, dass „Hersteller sich mehr denn je mit dem gesamten Lebenszyklus ihrer Produkte auseinander setzen müssen“ beziehungsweise „weitere Fortschritte nur durch die Zusammenarbeit von Wirtschaft, Politik und Konsumenten zu erreichen sind“. Die meisten Befragten erwarten von Umweltschutzkonzepten offenbar auch, dass ,die ganze Branche mitzieht" und „,besonders bei Produktentwicklern das Bewusstsein für eine ökologisch nachhaltige Entwicklung geschärft werden muss“.

Dass es nur mit Selbstverpflichtungen der Wirtschaft und ohne staatliche Regulierungen künftig Fortschritte im Umweltschutz geben werde, wird jedoch von der Mehrheit bezweifelt. Noch größere Zweifel bestehen, wenn zu viele Interessengruppen mitreden, wenn hauptsächlich der Nutzungsphase die Hauptschuld an Umweltschäden angelastet oder den Herstellern allein die Entscheidung über die Umweltverträglichkeit ihrer Produkte zugemutet wird. Stärker zustimmungsfähig waren dagegen wiederum
Statements, die sich auch für neue Konzepte aussprachen und die ein größeres Problem im Informationsmanagement von Daten, insbesondere zwischen Herstellern und Zulieferern, verorten. Vergleichsweise unstrittig ist auch die künftige Rolle des Verbrauchers. Etwa 70 Prozent der Befragten sind der Auffassung, dass er sich umweltbewusster verhalten müsse als bisher.

Insgesamt kann also im Jahr 2003 festgestellt werden, dass die einzelnen Inhalte der Integrierten Produktpolitik in der bayerischen produzierenden Wirtschaft von mindestens jedem fünften Befragten positiv eingeschätzt werden, wobei auf Ganzheitlichkeit, Kooperation und Nachhaltigkeit zielende Statements Zustimmung von über 80 Prozent aller Befragten erhielten (1).

\section{Anmerkung}

(1) Ausführliche Darstellungen der Ergebnisse stehen unter www. bifa.de/veroffent/index.html als BlfA Texte Nr. 19 und 28 zum kostenlosen Download zur Verfügung.

\section{Die Autoren}

Siegfried Kreibe ist stellvertretender Abteilungsleiter Umwelttechnik des Bayerischen Instituts für Angewandte Umweltforschung und -technik GmbH (BIfA). Michael Schneider ist Privatdozent am Lehrstuhl für Soziologie der Technischen Universität München. Kontakt: BIfA GmbH, Am Mittleren Moos 46, 86167 Augsburg. E-Mail: skreibe@bifa.de, TU München, Lehrstuhl für Soziologie, Lothstraße 17, 80335 München. E-Mail: Michael.Schneider@ws.tum.de 
(c) 20I0 Authors; licensee IÖW and oekom verlag. This is an article distributed under the terms of the Creative Commons Attribution Non-Commercial No Derivates License (http://creativecommons.org/licenses/by-nc-nd/3.o/), which permits unrestricted use, distribution, and reproduction in any medium, provided the original work is properly cited. 